\title{
Unterrichtsqualität ist immer generisch und fachspezifisch. Ein Kommentar aus kognitions- und lehr-lerntheoretischer Sicht
}

\author{
Kurt Reusser $\cdot$ Christine Pauli iD
}

Eingegangen: 28. Januar 2021 / Überarbeitet: 3. Mai 2021 / Angenommen: 11. Mai 2021 / Online publiziert: 9. Juni 2021

(C) Der/die Autor(en) 2021, korrigierte Publikation 2021

Zusammenfassung Orientierten sich Allgemeine Didaktik und Unterrichtsforschung lange Zeit fast ausschließlich an generischen Qualitätsmerkmalen von Unterricht, so werden heute zunehmend ebenfalls fachdidaktische Merkmale in den Blick genommen. Der Beitrag zeichnet zuerst einige Entwicklungslinien nach, die in den vergangenen Jahren zur Erkenntnis eines Bedarfs und zu einem wachsenden Interesse an einer fachdidaktisch ausdifferenzierten Unterrichtsforschung geführt haben. Danach werden eine Reihe von Theorieproblemen benannt, die wir als Desiderata einer fachdidaktisch konkretisierten Unterrichtsforschung für wichtig halten. Der Beitrag schließt mit der Skizzierung von Forschungs- und Entwicklungsaufgaben, die von den beteiligten Akteuren und Disziplinen nur in transdisziplinärer Zusammenarbeit gelöst werden können, und denen eine Stärkung empirischer Zugänge gemeinsam ist.

Schlüsselwörter Generische und fachdidaktische Unterrichtsqualität · (Fach-)Didaktik · Fachspezifität · Lehr-Lernforschung

Kurt Reusser

Institut für Erziehungswissenschaft, Universität Zürich, Zürich, Schweiz

E-Mail: reusser@ife.uzh.ch

Christine Pauli $(\bowtie)$

Zentrum für Lehrerinnen- und Lehrerbildung, Universität Fribourg, Fribourg, Schweiz 


\title{
Teaching quality is always generic and subject-specific. A commentary from the perspective of cognitive psychology and teaching-learning theory
}

\begin{abstract}
For a long time, general didactics and teaching research were almost exclusively oriented towards generic quality characteristics of teaching. Today, however, subject-didactic characteristics are increasingly being taken into account. The article first traces some lines of development that have led in recent years to the recognition of a need and to a growing interest in subject-didactically differentiated classroom research. Afterwards, a number of theoretical problems are named, which we consider important as desiderata of a subject-didactically differentiated classroom research. The article concludes by outlining research and development tasks that can only be solved by the participating actors and disciplines in transdisciplinary cooperation, and which have in common a strengthening of empirical approaches.
\end{abstract}

Keywords Teaching quality $\cdot$ Content specificity $\cdot$ Didactics $\cdot$ Generic and subjectspecific quality of instruction

\section{Unterrichtsqualität zwischen pädagogisch-didaktischer Theoriebildung und empirischer Lehr-Lernforschung}

Was die Qualität von Unterricht ausmacht, wird seit jeher von der (Allgemeinen) Didaktik und von den Fachdidaktiken, in neuerer Zeit und mit Blick auf die Erreichung von Bildungszielen von der empirischen Lehr-Lernforschung bearbeitet. Während die Allgemeine Didaktik als langjährige Referenzdisziplin zum Thema guter Unterricht in den letzten Jahrzehnten unter Druck geraten ist und ihre Bedeutung für die Lehrpersonenbildung abgenommen hat, haben die empirische Lehr-Lernforschung und die Fachdidaktik an Reputation zugelegt (Reusser 2008, 2018a, 2020).

Vor allem die pädagogisch-psychologische Unterrichtsforschung hat in den vergangenen Jahrzehnten einen veritablen Aufschwung erlebt. Zu erwähnen ist, dass es bereits vor der (ab den 1960er-Jahren einsetzenden) kognitiven Wende in der Psychologie eine bis an den Anfang des Jahrhunderts zurückreichende, mit Namen wie Meumann, Thorndike, Lay, Petersen, Dewey verbundene pädagogische Forschung gab, welche allgemeine Merkmale des Lehrerverhaltens wie z. B. die Klassenführung, den Erziehungsstil (seit Kurt Lewin) oder die Qualität der Lehrer-SchülerInteraktion (TauschTausch 1970) untersuchte (zfd. Depaepe 1993; Einsiedler 2000). Mit dem Aufstieg des kognitiv- und sozial-konstruktivistischen Lernverständnisses rückten zuerst im englischsprachigen (Bloom 1973; Caroll 1963), sodann auch im deutschsprachigen Raum immer stärker Qualitätsmerkmale des Unterrichtshandelns in den Fokus, die sich auf die Unterstützung von inhaltlichen Schülerlernprozessen und damit auf Fachunterricht bezogen. Als exemplarisch dazu ist die „Münchner Schulleistungsstudie“" (SCHOLASTIK-Studie) von Helmke et al. (1986) zu nennen, die durch die Operationalisierung zahlreicher Merkmale der Qualität von Unterricht (z.B. Klarheit/Strukturiertheit, Aktivitätsniveau der Klasse, individuelle Unterstüt- 
zung, Adaptivität) wesentlich dazu beigetragen hat, dass Unterrichtsqualität im deutschen Sprachraum zu einem eigenständigen Forschungsbereich geworden ist (vgl. Einsiedler 2000, für einen Überblick). Diese Entwicklung steht im Einklang mit der Überzeugung, dass sich die Prozesse des schulischen Lernens und seiner Anregung in ihrer Bedingtheit und Prozessqualität durch die psychologisch orientierte LehrLern-Forschung am besten rahmen und ausbuchstabieren lassen.

Obgleich es in empirischen Studien zur Unterrichtsqualität stets um gegenstandsbezogene Lehr-Lernprozesse in Fächern ging, wurden (insbesondere im Rahmen des Prozess-Produkt-Paradigmas) identifizierte Qualitätsmerkmale meist fachgeneralisierend - d. h. ohne Berücksichtigung von Fachspezifika - interpretiert. Als solche flossen sie in zahlreiche allgemeindidaktische Beschreibungen guten Unterrichts ein, wie z. B. in die aus der pädagogischen Tradition hervorgegangenen, durch Forschungsergebnisse präzisierten Listen etwa von Meyer (2004) oder Helmke (2009), bis hin zu den Meta-Analysen von Hattie (2009). Auch in den meisten Modellen der Allgemeinen Didaktik spielten fachlich-fachdidaktische Aspekte eine eher geringe Rolle. Eine Ausnahme bildete die bildungstheoretische Didaktik in der von Wolfgang Klafki vertretenen Ausprägung. Sein in der Lehrkräftebildung (kaum jedoch in der Lehr-Lernforschung) rezipierter, 1958 erstmals publizierter Aufsatz „Didaktische Analyse als Kern der Unterrichtsvorbereitung“ (Klafki 1958) legte den Akzent auf fachdidaktische Fragen, wie das Herausschälen des Bildungswertes eines Stoffes, seiner Gegenwarts- und Zukunftsbedeutung für die Bildung des Kindes und die Freilegung seiner bildenden (medialen) Zugänge. Trotz sich bietender Anknüpfungspunkte u.a. für die Klärung von (für fachliche Lernprozesse bedeutsamen) Fragen wie die bildungszielbezogene und niveaugerechte Aufbereitung von Stoffinhalten (Bruner 1970: ihre ,intellektuell redliche didaktische Reduktion“) und die Bestimmung der Qualität fachinhaltlicher Lerngelegenheiten (OTL), insbesondere der Potenziale von Lernaufgaben, wurde das Anregungspotenzial der bildungstheoretischen Didaktik von der empirischen Bildungsforschung kaum wahrgenommen.

Was die pädagogisch-psychologische Lehr-Lernforschung anlangt, begann sich ab den 1980er-Jahren die dazu den Kontext bildende Landschaft der kognitiven Entwicklungs- und Lerntheorie insofern zu verändern, als es zu einer Abkehr von allgemeinen (für alle kognitiven Phänomene und Funktionen geltenden) und universellen (Stufen-)Theorien der geistigen Entwicklung (z. B. sensu Piaget), und zu einer Hinwendung zu Theorien des bereichsspezifischen Wissensaufbaus (ExpertiseForschung) kam. Nachhaltig damit verbunden war die Entdeckung des Wissens als inhaltliche Seite der Intelligenz und des Kompetenzerwerbs bzw. die Aufwertung der Bedeutung eines intelligent strukturierten, bereichsspezifischen Inhaltswissens für den Vollzug anspruchsvoller geistiger Leistungen (Aebli 1980/1981; Reusser 1998, für einen Überblick). ${ }^{1}$ Zahlreiche Studien aus der Experten-Novizen-Forschung (Chi et al. 1988; Ericsson und Smith 1995) legten nahe, nicht nur herausragende Lernleistungen von Experten, sondern auch intra- und interindividuelle Leistungsunter-

\footnotetext{
1 Vgl. für den deutschsprachigen Raum den seminalen (von Hans Aebli in deutscher Sprache herausgegebenen) Band „Strukturen des Wissens. Wege der Kognitionsforschung“ von Norman und Rumelhart (1978) und den dadurch angeregten Aufschwung der sog. Wissenspsychologie in Theorie und Forschung, exemplarisch dokumentiert im Sammelband „Wissenspsychologie“ (Mandl und Spada 1988).
} 
schiede von Kindern sowie Unterschiede zwischen Kindern und Erwachsenen in den verschiedensten Sachgebieten als Ergebnis inhaltsspezifischer Wissensbildungsprozesse zu interpretieren. Die ebenfalls in diesem Zeitkontext zu sehenden, oftmals zitierten Texte von Shulman $(1986,1987)$ zu Begriff und Bedeutung des „Pädagogischen Inhaltswissens“ machen deutlich, dass dies auch für das professionelle Wissen und Handeln von Lehrpersonen gilt. Auch die Qualitäten des Lehrens sind nicht einfach allgemein pädagogischer Natur, sondern umfassen als ein komplexes Können nebst generischem pädagogisch-didaktischem auch fachdidaktisches bzw. fachspezifisch-pädagogisches Wissen (Shulman: ,that special amalgam of content and pedagogy that is uniquely the province of teachers, their own special form of professional understanding“, 1987, S. 8). Damit anspruchsvolles Lernen im Sinne des Erwerbs gegenstandsspezifischer Konzepte, Methoden und Denkformen im Unterricht gelingt, bedarf es des Zusammenspiels von zahlreichen, auf unterschiedlichen Ebenen angesiedelten Qualitäten des Professionswissens (Kunter et al. 2011). Die Einsicht in die Notwendigkeit, das Zusammenwirken von generischen und fachspezifischen Merkmalen besser zu verstehen und diesem auch in der Ausbildung von Lehrpersonen Rechnung zu tragen, hat nicht nur die fachdidaktische Unterrichtsforschung zunehmend befruchtet (vgl. COACTIV) und zu einer verstärkten Erforschung der professionellen Kompetenz von Lehrpersonen aus einer fachlichen Perspektive geführt, sondern auch die fachdidaktische Lehrpersonenbildung beeinflußt (Staub 2001).

Wie wichtig das Zusammenspiel von generischen und fachspezifischen Unterrichtsmerkmalen für den Lernfortschritt und die Leistungsentwicklung sind, verdeutlicht die Metaanalyse von Seidel und Shavelson (2007). In der Studie erwiesen sich fachspezifische Lernaktivitäten (,domain-specific learning activities“) fach- und stufenunabhängig als erklärungsstärkste Komponente für den Nachweis der Effektivität von Lehr-und Lernprozessen. Als Beispiele solcher Lernaktivitäten werden darin ,mathematical problem solving, scientific inquiry“ und „specific reading and writing strategies“" genannt (ebd., S. 470).

Die Ergebnisse des Forschungsüberblicks sind für die Frage nach der Fachspezifität von Unterrichtsqualität in zweierlei Hinsicht bedeutsam. Zum einen illustrieren sie, dass Qualitätsmerkmale sowohl fachübergreifend (generisch) als auch fachspezifisch konzipiert werden können, je nachdem, auf welcher Abstraktions-oder Generalisierungsebene sie betrachtet werden. Dies gilt auch für die sog. Basisdimensionen von Unterrichtsqualität (Klieme et al. 2006) und das darauf bezogene „Syntheseframework“ von Praetorius et al. (2020a). So zeigen sich auf der Ebene der Subdimensionen, und noch stärker auf der Ebene der Kriterien, jeweils mehr oder weniger stark voneinander abweichende Merkmalsbeschreibungen der entsprechenden Basisdimension, bezogen auf die Fächer Naturwissenschaft (Heinitz und Nehring 2020), Sport (Herrmann und Gerlach 2020) und Geschichte (Zülsdorf-Kersting 2020). Zum andern unterstreichen die Ergebnisse die grundsätzliche Bedeutung fachspezifischer Aspekte von Unterrichtsqualität bzw. der Umsetzung generischer Merkmalsdimensionen. Das Herausstellen der wichtigen Bedeutung der Lerninhalte steht für eine Weiterentwicklung, die als fachdidaktische Wende in der aktuellen empirischen Lehr-Lernforschung bezeichnet werden kann. Ähnlich wie die im Verhältnis zur Allgemeinen Didaktik einflussreicher gewordene Lehr-Lernforschung 
zu einem Theorieschub hinsichtlich einer empirisch gestützten Konzeptualisierung generischer Basisdimensionen geführt hat, sind durch die Ausdifferenzierung der fachdidaktischen Forschung Erkenntnisfortschritte mit Bezug auf ein tieferes Verständnis der fachspezifischen Qualität von Unterricht zu erwarten.

\section{Fachdidaktische Unterrichtsqualität als Ausdifferenzierung eines globalen Kerns von allgemein didaktischen Merkmalen}

Unterricht als zwischen „Gegenstand“, „Schülergruppe“ und „Lehrperson“ angesiedelter, interaktiver Wissens- und Kompetenzaufbau ist immer Fachunterricht (Reusser 2008, 2018a). So wie der menschliche Erkenntnisweg „über die Dinge“ führt, bedeutet auch Unterricht sich auf Stoffe und Gegenstände einzulassen. Seine Qualität zu erforschen, sich inhaltlich mit dessen konstitutiver Gerichtetheit auf materielle, kulturelle, soziale und geistige Gegenstände, d.h. mit seiner fachlichen Qualität zu beschäftigen. Unterrichtsinhalte und Bildungsziele in ihrer aufbereiteten Sachstruktur müssen dabei ebenso thematisiert werden wie deren sachlogische und psychologische Erschliessungs- und Aufbauqualität und die damit verbundenen, angebotsund nutzungsseitigen Aneignungsprozesse und -herausforderungen. Auch wenn Unterricht per definitionem Fachunterricht ist, ist dessen Bildungszielsetzung keine additiv-einzelfachliche. Angestrebt wird eine fachliche Allgemeinbildung (Literacy) im Sinne eines Ensembles von unterschiedlichen „Modi der Weltbegegnung“ verbunden mit basalen sprachlichen, mathematik- und selbstregulationsbezogenen Kulturkompetenzen (Baumert 2002). Bei aller inhaltlichen Verschiedenheit der Unterrichtsfächer weisen diese dennoch zahlreiche Gemeinsamkeiten auf. Noch mehr gilt dies für die von der pädagogisch-psychologischen Forschung beschriebenen Bedingungen und Prozesse für eine erfolgreiche Aneignung von Wissen und Können in ganz unterschiedlichen Inhalts- und Lernbereichen. Wenn in der Unterrichtsqualitätsforschung sich in den letzten Jahren ein Kern generischer Basisdimensionen als erklärungsstark für die Entwicklung von Wissen und Verstehen erwiesen haben, dann weil sie allgemeinpsychologische Merkmale von Lern- und Entwicklungsprozessen widerspiegeln. Als ein weiterer kardinaler Punkt kommt hinzu, dass Unterricht keine ausschließlich fachlich-epistemische, sondern ebenfalls eine pädagogische Zielsetzung verfolgt. Das heißt, dass im Unterricht auch persönlichkeitsbildende (überfachliche) Kompetenzziele angestrebt werden, deren Erreichung mit Qualitätsmerkmalen zusammenhängt, die zwar auch im Fachunterricht ausgebildet werden, jedoch selbst nicht bloss fachlicher Natur sind.

\subsection{Videostudien als Katalysator einer (fach)didaktisch konkretisierten Unterrichtsforschung}

Kaum ein Forschungstyp hat als Quelle der Anregung das Nachdenken über Bildungs- und Unterrichtsqualität in den letzten Jahrzehnten mehr befruchtet und ist zu einem Treiber nicht nur der generischen pädagogisch-psychologischen, sondern in jüngerer Zeit auch einer fachdidaktisch sich ausdifferenzierenden Unterrichtsforschung geworden wie die videobasierte Forschung, welche mit den beiden TIMSS- 
Videostudien 1995 und 1999 (Hiebert et al. 2003; Stigler et al. 2000) ihren Anfang nahm. Das Potenzial von Videodaten liegt darin, dass Unterricht raum-zeitlich unabhängig und durch unterschiedliche, auch interdisziplinäre Forschungsteams anhand großer Stichproben mikroanalytisch, theoretisch und methodisch mehrperspektivisch untersucht werden kann (Pauli und Reusser 2006; Reusser und Pauli 2013). So wurde bereits in der ersten TIMSS Videostudie, die sich auf die Länder USA, Japan und Deutschland bezog, die kognitiv-fachliche Anforderungsqualität der in Vertiefungsphasen gelösten Aufgaben aus einer fachdidaktischen Perspektive analysiert (Neubrand 2004). Eine zentrale Rolle spielten fachspezifische Unterrichtskomponenten und fachdidaktische Gesichtspunkte (u.a.: „Aufgaben“ als zentrales Gliederungsmerkmal) auch in der zweiten TIMSS Videostudie (Hiebert et al. 2003), an der von Anfang an ein fachdidaktisches Expertenteam mitbeteiligt war.

Videodaten erlauben die Analyse einer breiten Palette von für zahlreiche Fächer bedeutsamen, allgemeinen und gegenstandsspezifischen Qualitätsmerkmalen von Unterricht vor dem Hintergrund eines breiten Spektrums von (über)fachlichen Bildungszielen und Unterrichtsformen. Dazu gehören Merkmale wie ,Aufgabenqualität“, ,kognitive Aktivierung“, ,konstruktive Unterstützung“ oder ähnlich schwierig erfassbare Merkmale wie z.B. ,Verständnisklarheit in der Präsentation von Inhalten“, „dialogische Gesprächsführung“ oder motivational-emotionale Unterstützung. Darüber hinaus wird es möglich, Zusammenhänge, Überschneidungen und Unterschiede zwischen generisch und fachspezifisch formulierten Qualitätskriterien zu untersuchen.

Dass fachdidaktisch ausgerichtete Videoanalysen dabei besonders ertragreich sind, zeigen beispielsweise die Analysen der Pythagoras-Videostudie (Klieme et al. 2009). Während sich in der Studie nur geringe Zusammenhänge zwischen den generisch gefassten Ratings der ,kognitiven Aktivierung“ und jenen der ,fachdidaktischen Qualität“ im Sinne der Anregung von konzeptspezifischen Verknüpfungen (Drollinger-Vetter 2011) zeigten, erwies sich die „fachdidaktische Qualität“ als stärkster Prädiktor für die konzeptspezifische Verständnisentwicklung. Weiter zeigte sich für die Dimension der kognitiven Aktivierung in den videobasierten Ratings die höchste Variabilität bzw. die vergleichsweise geringste Stabilität, was ebenfalls auf deren Inhaltsabhängigkeit hindeutet (Praetorius et al. 2014). Die Befunde sprechen für eine fachspezifische Konkretisierung der Dimension der ,kognitiven Aktivierung", insbesondere mit Bezug auf Aspekte, die die Qualität, Vollständigkeit und Art der inhaltsspezifischen Impulsgebung und der Stoffbearbeitung betreffen (Lipowsky et al. 2018). Da videobasierte Datenerhebungen in quantitativ und qualitativ ausgerichteten fachdidaktischen Forschungsprojekten heute omnipräsent sind, dürften mehrperspektivische und multimethodische Videoanalysen unter Einbezug der dem Unterricht zugrundeliegenden Materialien und daraus hervorgegangenen Artefakten, verstärkt insbesondere durch Fallanalysen, den Blick auf die fachspezifischen Mikrostrukturen von Unterrichtsqualität nachhaltig weiter schärfen.

Auf der Grundlage einer internationalen Synthese von generischen und fachspezifischen Merkmalen der Unterrichtsqualität im (privilegiert untersuchten) Fach Mathematik haben Praetorius et al. (2020a) und Praetorius et al. (2020b) den Forschungsstand zur Unterrichtsqualität für vier weitere Fachdidaktiken analysiert und Desiderate für fachspezifische Anpassungen und Ergänzungen identifiziert. Es zeigte 
sich, dass insbesondere auf der Ebene der Subdimensionen des zugrunde liegenden „Syntheseframeworks“, und noch stärker auf der Ebene der diesen zugeordneten Indikatoren - fachbezogene Ausdifferenzierungen erforderlich sind.

\subsection{Desiderate der fachdidaktischen Theoriebildung}

Teilt man auf Basis des Diskussionsstandes die Einschätzung, dass in zahlreichen Fächern eine empirisch-systematische fachdidaktische Unterrichtsqualitätsforschung noch eher am Anfang steht, so lassen sich insbesondere bezüglich der fachspezifischen Ausdifferenzierung eines globalen Konzeptes bzw. generischer Merkmale von Unterrichtsqualität eine Reihe von Problemen identifizieren, die wir nicht nur als Theorieprobleme für bedeutsam, sondern auch für die Entwicklung fachdidaktischer Kodier- und Messinstrumente als wichtig erachten.

1. Der Bedarf nach fachspezifischer Ergänzung und Ausdifferenzierung von globalen Kerndimensionen ist von Fach zu Fach, und innerhalb eines Fachs von Teilkompetenz zu Teilkompetenz verschieden. Fächer repräsentieren unterschiedliche Wissensdomänen und Denkkulturen. Betrachtet man Disziplinen aus einer epistemischen Perspektive, bestehen sie in der Regel aus einem identifizierbaren Korpus von Begriffen und Ideen, einer Syntax zu deren Verknüpfung, charakteristischen Formen der Etablierung von Wahrheit, Evidenz und Verständnisqualität, sowie mehr oder weniger besonderen, durch die Stoffe und Gegenstände bestimmten wissenschaftlichen Methoden des Untersuchens und Forschens (Bruner 1970; Hirst 1974). Die daraus sich ergebenden Wissens- und Denkformen von Fachdisziplinen pädagogisch zu kultivieren erfordert deshalb nebst der fachlichen Begriffsbildung die Ausbildung von spezifischen Formaten der Wissensrepräsentation, Methoden und Diskursformen. Den Satz des Pythagoras zu beweisen ist nicht dasselbe wie in der Grundschule die Schriftsprache zu lehren oder Kindern das Lesen beizubringen, in den Naturwissenschaften das Springen eines Gummiballs vor dem Hintergrund mitgebrachter Fehlkonzepte verstehbar zu machen, in der Geschichte einen historischen Quellentext gemeinsam mit der Klasse kontextsensibel zu interpretieren oder in einem Lerndialog das moralisch implizierte Handeln einer Person in einer Dilemma-Situation mehrperspektivisch zu erörtern und zu beurteilen. Um solcher Verschiedenheit nicht nur in der fachdidaktischen Unterrichtspraxis, sondern auch in der Forschung gerecht zu werden, braucht es neben der kognitiven Analyse fachlicher Wissens- und Denkformen die Bearbeitung von Fragen der Inhaltsqualität, Auswahl, Sequenzierung und Aufbereitung von Unterrichtsinhalten. Solche Fragen müssen stärker als bisher in einen Theoriekern der Erforschung von Unterrichtsqualität und professioneller Kompetenz integriert werden. Wie weit sich ausdifferenzierte Facetten fachlicher Unterrichtsqualität auf andere Fächer bzw. Gegenstände übertragen lassen, muss als empirische Frage behandelt und untersucht werden.

2. Je näher an konkreten Gegenständen und schulformspezifischen Anforderungen die Qualität von Lehr-Lernprozessen bestimmt werden soll, desto zwingender ist die inhaltsspezifische, d.h. gegenstandsnahe, empirische Analyse ihrer lehrseitigen Aktivierungs- und Unterstützungsanforderungen sowie der schülerseitig zu 
erwartenden Verständnisherausforderungen samt der zu ihrer Bewältigung notwendigen geistigen Operationen. Für zahlreiche (insbesondere naturwissenschaftliche) Konzepte bedeutet dies ebenfalls den Einbezug von Conceptual-ChangeForschung zu Fehlkonzepten und zu Stufen und Klippen des Erwerbs wissenschaftlicher Begriffe. Des Weiteren dürfte für die globalen Merkmale gelten, dass der Bedarf nach fachdidaktischer Detaillierung umso ausgeprägter ist, je leistungsschwächer die Schüler/innen bzw. je höher die von ihnen wahrgenommene Aufgabenschwierigkeit bzw. Anforderung ist.

3. Der Bedarf nach fachlicher Ausdifferenzierung von Merkmalen ist kein ",feststehender", sondern hängt von zu beantwortenden pädagogisch-psychologischen und didaktischen Forschungsfragen $a b$. Geht es z. B. darum, sich auf einer fachgeneralisierenden (fachneutralen) Schulebene ein Bild von der Qualität des Unterrichts zu machen, dürfte der Bedarf nach Ausdifferenzierung einer Qualitätsdimension geringer sein, als wenn die Qualität gegenstandsbezogener Lehr-Lernprozesse auf Individual- oder Klassenebene untersucht werden soll. Das heißt, dass je nach Ziel und Nutzungskontext (z. B. Aus- und Weiterbildung von Lehrpersonen; Entwicklung von Lehrmitteln oder Unterrichtsbausteinen) der Bedarf nach Spezifizierung bzw. der Grad der notwendigen Auffaltung („Auflösung“; Lindmeier und Heinze 2020) des Konstrukts Unterrichtsqualität und seiner Dimensionen ein anderer ist.

4. Eine fachspezifische Ausdifferenzierung erfordern primär jene Unterrichtsqualitätsmerkmale, deren Fokus ein epistemischer ist, d.h. die sich auf die kognitiven Prozesse des Aufbaus, der Integration, Umstrukturierung, Erweiterung und Differenzierung konzeptspezifischer Verknüpfungen richten. Bereits 1913 hat der Denkpsychologe Otto Selz, einer der Urväter der kognitiven Psychologie, von „Sachverhältnissen“ als den Strukturbausteinen unseres Wissens und Denkens gesprochen (Selz 1913): „Sachverhältnisse sind das in einer bestimmten Beziehung Stehen bestimmter Gegenstände“. Da mannigfaltige und gleichzeitig spezifische Sachbeziehungen allen disziplinären Denk- und Lernprozessen zugrunde liegen und deren gegenstandsspezifische Wissensstrukturen konstituieren, können diese auch nicht bloß allgemein - in der damaligen Sprache der Psychologie: ,assoziativ“ - sondern nur fachspezifisch wirksam angeregt werden. Ganz besonders trifft dies auf das derzeit meistdiskutierte generische Merkmal der kognitiven Aktivierung zu, das sich auf die Anregung von Verknüpfungen der zentralen Verstehenselemente bezieht, die zur Bildung von Fachbegriffen und zur Erklärung, Präzisierung, Erörterung, Differenzierung von Sachverhalten notwendig sind. Die hier nur angedeutete Position, die auch Aebli in seiner denkpsychologisch fundierten Didaktik (Aebli [1980] 1981, 1983) vertreten hat, ist ebenfalls in unsere Pythagoras-Studie eingeflossen (Drollinger-Vetter 2011; Lipowsky et al. 2018).

5. Nicht nur beim repräsentational mehrstufigen Aufbau deklarativen Wissens, sondern auch bei der Vermittlung von Regulations- und Lernstrategien spielt die Spezifität kognitiv aktivierender und unterstützender Denkanstöße, Impulse und Prompts (Glogger et al. 2009; Schoenfeld 1985) eine wichtige Rolle im Unterricht. In eine ähnliche Richtung weist auch die Forschung zu formativ-diagnostischer Lernunterstützung (formative Assessment), „Scaffolding“ und adaptivem Feedback in seinen diversen Formen und Funktionen (Bürgermeister und Saalbach 
2018; Hattie und Timperley 2007; Reusser 2018b; William 2015). Kognitiv aktivierendes und adaptiv unterstützendes Lehrerhandeln erweist sich vor allem dann als hilfreich, wenn es diagnostisch gehaltvoll und gegenstandsspezifisch ist, nahe an den Denkprozessen der Lernenden erfolgt, und an die Lernvoraussetzungen insbesondere von leistungsschwächeren Kindern anknüpft (Hahn et al. 2016). Umgekehrt ist mit Bezug auf die Kompetenz von Lehrpersonen davon auszugehen, dass Lehrkräfte mit einem hohen fachlich-fachdidaktischen Wissen (Pädagogisches Inhaltswissen sensu Shulman 1986) eher zu einer hochwertigen Impulsgebung mit Bezug auf die Anregung einer vertieften Auseinandersetzung mit Bildungsgegenständen und zu einem präzisen „feed forward“ (sensu Hattie) einschließlich der Identifikation von auftretenden Verständnisklippen fähig sind als Lehrpersonen mit einem geringen Wissen.

6. Ein weiteres Globalmerkmal qualitätsvollen Lehrkräftehandelns mit einer hohen fachspezifischen Aufladung, dem aktuell eine wachsende Aufmerksamkeit zukommt, dessen fachdidaktische Seite jedoch noch unzureichend - sowohl phänomenologisch als auch empirisch-analytisch - ausgeleuchtet ist, betrifft die $\mathrm{Kul}$ tivierung der Fähigkeit zur Führung gegenstandsbezogener, dialogischer Unterrichtsgespräche bzw. zur verantwortlichen Teilnahme der Schülerinnen und Schüler am Unterrichtsdiskurs (Heller und Morek 2019; Resnick et al. 2018). In der Studie Socrates 2.0 (Pauli und Reusser 2018) wurden Merkmale eines diskursiven Unterrichts in den Fächern Mathematik und Geschichte herausgearbeitet. Danach wurden Lehrpersonen geschult, mit der Klasse dialogische Unterrichtsgespräche zu anspruchsvollen Themen zu führen. Es konnte gezeigt werden, dass sich die Qualität der Unterrichtsgespräche sowohl in Bezug auf die Partizipationsstrukturen als auch in Bezug auf den fachlichen Gehalt steigern liess. Eine wesentliche Rolle spielten dabei ein erweitertes Repertoire an fachübergreifenden und fachspezifischen Strategien des Aufgreifens und Weiterentwickelns von Schülerbeiträgen und der Gesprächssteuerung sowie fachübergreifende und fachspezifische Merkmale des Gesprächskontextes (u.a. Aufgabenqualität, Ressourcen, Vorbereitung; Zimmermann 2020).

7. Wo fachlich-fachdidaktische Spezifizierungen eine möglicherweise etwas geringere Rolle spielen dürften, ist bei Qualitätsmerkmalen, die sich auf die soziale Dimension der Klassenführung, die Aufrechterhaltung eines positiven Lernklimas in seiner Grundschicht (Wohlbefinden) oder auf allgemeine Aspekte der motivationsförderlichen Qualität von Unterricht (z.B. Autonomieunterstützung, soziale Eingebundenheit) beziehen. Allerdings sollte diese Einschätzung ebenfalls auf den Prüfstand der empirischen Forschung gestellt werden. Mutmaßlich gilt auch hier, dass je mehr man sich gegenstandsbezogenen Kompetenzanforderungen quasi mit dem Brennglas nähert, desto wahrscheinlicher auch hier fachspezifische Differenzen dennoch zutage treten dürften. So bedeutet z. B. Klassenführung im Sportunterricht wohl nicht unter allen Subdimensionen des Konstrukts exakt dasselbe wie in anderen Fächern. 


\section{Zum Selbstverständnis einer empirisch ausgerichteten, fachdidaktischen Bildungsforschung und zur Kooperation zwischen den Disziplinen - ein Ausblick}

Was braucht es, um die Bedeutung und die Wirkungsweise von fachdidaktischen Merkmalen der Unterrichtsqualität im Zusammenspiel mit einem Kern globaler Qualitätsdimensionen langfristig noch besser zu verstehen? Dies zu einen mit Bezug auf die Erforschung und Messung fachdidaktischer Lehr-Lernqualität, zum andern mit Bezug auf deren Förderung im Unterricht einschließlich der Vorbereitung dazu in der Lehrkräftebildung. In den nachfolgenden Schlussbemerkungen plädieren wir für eine Stärkung der empirischen Zugänge in den beteiligten Disziplinen und für eine engere Zusammenarbeit zwischen (Fach)Didaktik(en) und empirischer Bildungsforschung.

Kognitionswissenschaftlich orientierte Fachdidaktik. In allen Fächern besteht Entwicklungsbedarf bei der kognitiven Analyse von gegenstandsbezogenen LehrLernprozessen Damit die schülerseitigen Anforderungen an fachliche Sinnbildungsprozesse bzw. die Ausbildung elementarer Wissens- und Denkkompetenzen, und die damit verbundenen, lehrseitigen Herausforderungen besser verstanden werden können, braucht es die Analyse von Schlüsselkonzepten, Wissensformen und -zugängen, fachspezifischen Fähigkeiten und Denkformen und der damit verbundenen geistigen Aufbauprozesse. Eine Orientierung an der kognitionswissenschaftlichen Forschung ist deshalb hilfreich, weil letztere hinreichend präzise Begriffswerkzeuge für die Konzeptualisierung und Rahmung sowohl der Ordnungen der Sachwelt als auch der damit verbundenen geistigen Aufbauprozesse in sehr verschiedenen Realitäts- bzw. Literalitätsbereichen bereitstellt (vgl. Aebli 1980/1981 sowie die Ausführungen zu Desideratum 4). Fragen, die eine solche (nicht normative), empirisch-kognitionspsychologisch fundierte ,neue Stoffdidaktik“ beantworten soll, sind:

- In welcher Stufung und durch das Zusammenspiel welcher spezifischen konzeptuellen Elemente und Verknüpfungen entwickelt sich das Verständnis prototypischer fachlicher Konzepte?

- Welche Hürden und Verständnisherausforderungen stellen sich beim Aufbau von Begriffen und beim Lösen von fachlichen Aufgaben bei Schülerinnen und Schülern mit unterschiedlichen Lernvoraussetzungen?

- Was folgt daraus für die Qualität von deren fachspezifischer - kognitiv aktivierenden, dialogisch-diskursiven - Unterstützung im Unterricht inkl. Design von differenzierenden Aufgaben und Scaffolding-Hilfen, bezogen auf Grundformen und Standardaufgaben des Lehrens (Aebli 1983)?

Nicht alle Fachdidaktiken befinden sich am selben Ort, um sich auf die kognitionswissenschaftliche Modellierung ihrer Wissens- und Denkformen einzulassen einzulassen. Während sich einige Fachdidaktiken (darunter die Mathematik- und die Naturwissenschaftsdidaktik, vermehrt auch die Geschichtsdidaktik) in ihrer Forschung seit längerem an lern- und kognitionspsychologischen Konzepten und Me- 
thoden in der Analyse fachbezogener Lehr-Lernprozesse orientieren, ist dies für andere Fachdidaktiken weit weniger der Fall.

Weiterentwicklung der Fachdidaktiken zu empirisch forschenden Disziplinen Bei aller Unterschiedlichkeit in der Ausrichtung und Dynamik, mit der sich die Fachdidaktiken weiterentwickeln, ist der Trend hin zu ihrer fortschreitenden Professionalisierung im Spannungsfeld zwischen schulpraktischen und forschungsbezogenen Erwartungen, zwischen der Wahrnehmung von stoffdidaktischen Entwicklungsaufgaben und der Etablierung von Forschungszugängen allgegenwärtig. Die grundsätzlich zwischen Erkenntnis- und Entwicklungsorientierung anzusiedelnde fachdidaktische Forschung dürfte damit immer profilierter sowohl zum tieferen Verständnis der fachdidaktischen Prozessqualität von Unterricht (Klieme 2019; Klieme und Rakoczy 2008; Prediger et al. 2012) als auch zur wechselseitigen Durchdringung von generischen und fachdidaktischen Qualitätsmerkmalen beitragen.

Transdisziplinäre Zusammenarbeit $\mathrm{Zu}$ einem wissenschaftlich reflektierten Verständnis von Unterrichtsqualität tragen zahlreiche Disziplinen mit ihren je unterschiedlichen Erklärungsebenen bei. Diese Perspektiven gilt es als Zugänge und Sichtweisen zu integrieren. Die Aufgabe der Entwicklung eines disziplinübergreifenden Grundverständnisses von Unterrichtsqualität wird nur dann gelingen, wenn insbesondere die unterschiedlichen Traditionen und Methoden verpflichteten Disziplinen der (Fach)Didaktik(en) und der empirischen Lehr-Lern-Forschung enger als bisher zusammenarbeiten. Es gibt mittlerweile eine ganze Reihe von Forschungsprojekten, die dazu als Referenz dienen können, weil ihre Erträge sich für beide Seiten als positiv erwiesen haben. Zufolge der Heterogenität und der Fragmentierung nicht nur der fachdidaktischen, sondern auch der erziehungswissenschaftlichen Theorie- und Forschungslandschaft dürfte es allerdings noch länger dauern, bis unser Verständnis des Zusammenspiels von allgemeindidaktisch-lernpsychologischen (generischen) und fachspezifischen Qualitätsdimensionen von Unterricht einen Stand erreicht, der befriedigt und der sich ebenfalls in kohärenteren Konzepten der Ausbildung von Lehrpersonen aller Stufen und Fächer niederschlägt.

Funding Open Access funding provided by Université de Fribourg.

Open Access Dieser Artikel wird unter der Creative Commons Namensnennung 4.0 International Lizenz veröffentlicht, welche die Nutzung, Vervielfältigung, Bearbeitung, Verbreitung und Wiedergabe in jeglichem Medium und Format erlaubt, sofern Sie den/die ursprünglichen Autor(en) und die Quelle ordnungsgemäß nennen, einen Link zur Creative Commons Lizenz beifügen und angeben, ob Änderungen vorgenommen wurden.

Die in diesem Artikel enthaltenen Bilder und sonstiges Drittmaterial unterliegen ebenfalls der genannten Creative Commons Lizenz, sofern sich aus der Abbildungslegende nichts anderes ergibt. Sofern das betreffende Material nicht unter der genannten Creative Commons Lizenz steht und die betreffende Handlung nicht nach gesetzlichen Vorschriften erlaubt ist, ist für die oben aufgeführten Weiterverwendungen des Materials die Einwilligung des jeweiligen Rechteinhabers einzuholen.

Weitere Details zur Lizenz entnehmen Sie bitte der Lizenzinformation auf http://creativecommons.org/ licenses/by/4.0/deed.de. 


\section{Literatur}

Aebli, H. (1980/1981). Denken, das Ordnen des Tuns. Stuttgart: Klett-Cotta. Zwei Bände.

Aebli, H. (1983). Zwölf Grundformen des Lehrens. Stuttgart: Klett-Cotta.

Baumert, J. (2002). Deutschland im internationalen Bildungsvergleich. In M. Killius, J. Kluge \& L. Reisch (Hrsg.), Die Zukunft der Bildung (S. 100-150). Frankfurt a.M.: Suhrkamp.

Bloom, B.S. (1973). Individuelle Unterschiede in der Schulleistung: ein überholtes Problem? In W. Edelstein \& D. Hopf (Hrsg.), Bedingungen des Bildungsprozesses (S. 251-270). Stuttgart: Klett.

Bruner, J.S. (1970). Der Prozess der Erziehung. Berlin: Schwann. engl. Original 1960

Bürgermeister, A., \& Saalbach, H. (2018). Formatives Assessment. Ein Ansatz zur Förderung individueller Lernprozesse. Psychologie in Erziehung und Unterricht, 65, 194-205.

Caroll, J. B. (1963). A model of school learning. Teachers College Records, 64, 723-733. Deutsche Übersetzung: Ein Modell schulischen Lernens. In W. Edelstein \& D. Hopf (Hrsg.), Bedingungen des Bildungsprozesses (S. 234-250). Stuttgart: Klett.

Chi, M.T.H., Glaser, R., \& Farr, M. J. (Hrsg.). (1988). The nature of expertise. Hillsdale: Erlbaum.

Depaepe, M. (1993). Zum Wohl des Kindes? Pädologie, pädagogische Psychologie und experimentelle Pädagogik in Europa und den USA, 1890-1940. Weinheim: Deutscher Studien-Verlag.

Drollinger-Vetter, B. (2011). Verstehenselemente und strukturelle Klarheit. Fachdidaktische Qualität der Anleitung von mathematischen Verstehensprozessen im Unterricht. Münster: Waxmann.

Einsiedler, W. (2000). Von Erziehungs- und Unterrichtsstilen zur Unterrichtsqualität. In M. K. W. Schweer (Hrsg.), Lehrer-Schüler-Interaktion. Pädagogisch-psychologische Aspekte des Lehrens und Lernens in der Schule (S. 109-128). Opladen: Leske + Budrich.

Ericsson, K. A., \& Smith, J. A. (Hrsg.). (1995). Towards a general theory of expertise. Cambridge: Cambridge University Press.

Glogger, I., Holzäpfel, L., Schwonke, R., Nückles, M., \& Renkl, A. (2009). Activation of learning strategies in writing learning journals. Zeitschrift für Pädagogische Psychologie, 23(2), 95-104.

Hahn, E., Rohlfs, C., Wacker, A., \& Bohl, T. (2016). Umgang mit Heterogenität. Eine quantitative Beobachtungsstudie zur aktiven Lernzeit von Schülerinnen und Schülern unterschiedlicher Leistungsniveaus. In T. Bohl \& A. Wacker (Hrsg.), Die Einführung der Gemeinschaftsschule in Baden-Württemberg. Abschlussbericht der wissenschaftlichen Begleitforschung (WissGem) (S. 255-274). Münster: Waxmann.

Hattie, J. (2009). Visible learning. A synthesis of over 800 meta-analyses relating to achievement. London: Routledge.

Hattie, J., \& Timperley, H. (2007). The power of feedback. Review of Educational Research, 77(1), 88-112.

Heinitz, B., \& Nehring, A. (2020). Kriterien naturwissenschaftsdidaktischer Unterrichtsqualität - ein systematisches Review videobasierter Unterrichtsforschung. Unterrichtswissenschaft, 48(3), 319-360. https://doi.org/10.1007/s42010-020-00074-8.

Heller, V., \& Morek, M. (2019). Fachliches und sprachliches Lernen durch diskurs(erwerbs)orientierte Unterrichtsgespräche. Empirische Evidenzen und Desiderata mit Blick auf inklusive Settings. Didaktik Deutsch, 24(46), 102-121.

Helmke, A. (2009). Unterrichtsqualität und Lehrerprofessionalität. Diagnose, Evaluation und Verbesserung des Unterrichts. Seelze-Velber: Kallmeyer/Klett.

Helmke, A., Schneider, W., \& Weinert, F.E. (1986). Quality of instruction and classroom learning outcomes. Teaching and Teacher Education, 2, 1-18.

Herrmann, C., \& Gerlach, E. (2020). Unterrichtsqualität im Fach Sport - Ein Überblicksbeitrag zum Forschungsstand in Theorie und Empirie. Unterrichtswissenschaft, 48(3), 361-384. https://doi.org/10. 1007/s42010-020-00080-w.

Hiebert, J., Gallimore, R., Garnier, H., Givvin, K.B., Hollingsworth, H., \& Jacobs, J. (2003). Teaching mathematics in seven countries. Results from the TIMSS 1999 video study. Washington, DC: U.S. Department of Education, National Center for Education Studies..

Hirst, P.H. (1974). Knowledge and the curriculum. A collection of philosophical papers. London: Routledge and Kegan Paul.

Klafki, W. (1958). Didaktische Analyse als Kern der Unterrichtsvorbereitung. Die Deutsche Schule, 50(10), 450-471.

Klieme, E. (2019). Unterrichtsqualität. In M. Harring, C. Rohlfs \& M. Gläser-Zikuda (Hrsg.), Handbuch Schulpädagogik (S. 393-408). Münster: utb/Waxmann.

Klieme, E., \& Rakoczy, K. (2008). Empirische Unterrichtsforschung und Fachdidaktik. Zeitschrift für Pädagogik, 54(2), 222-237. 
Klieme, E., Lipowsky, F., Rakoczy, K., \& Ratzka, N. (2006). Qualitätsdimensionen und Wirksamkeit von Mathematikunterricht. Theoretische Grundlagen und ausgewählte Ergebnisse des Projekts „Pythagoras“. In M. Prenzel \& L. Allolio-Näcke (Hrsg.), Untersuchungen zur Bildungsqualität von Schule. Abschlussbericht des DFG-Schwerpunktprogramms (S. 127-146). Münster: Waxmann.

Klieme, E., Pauli, C., \& Reusser, K. (2009). The pythagoras study: investigating effects of teaching and learning in Swiss and German mathematics classrooms. In T. Janik \& T. Seidel (Hrsg.), The power of video studies in investigating teaching and learning in the classroom (S. 137-160). Münster: Waxmann.

Kunter, M., Baumert, J., Blum, W., Klusmann, U., Krauss, S., \& Neubrand, M. (Hrsg.). (2011). Professionelle Kompetenz von Lehrkräften. Ergebnisse des Forschungsprogramms COACTIV. Münster: Waxmann.

Lindmeier, A., \& Heinze, A. (2020). Die fachdidaktische Perspektive in der Unterrichtsqualitätsforschung: (bisher) ignoriert, implizit enthalten oder nicht relevant? Zeitschrift für Pädagogik, 66., 255-268. Beiheft.

Lipowsky, F., Drollinger-Vetter, B., Klieme, E., Pauli, C., \& Reusser, K. (2018). Generische und fachdidaktische Dimensionen von Unterrichtsqualität - Zwei Seiten einer Medaille? In M. Martens, K. Rabenstein, K. Bräu, M. Fetzer, H. Gresch, I. Hardy \& C. Schelle (Hrsg.), Konstruktion von Fachlichkeit. Ansätze, Erträge und Diskussionen in der empirischen Unterrichtsforschung (S. 183-202). Bad Heilbrunn: Klinkhardt.

Mandl, H., \& Spada, H. (Hrsg.). (1988). Wissenspsychologie. München: PVU.

Meyer, H. (2004). Was ist guter Unterricht? Berlin: Cornelsen Verlag Scriptor.

Neubrand, J. (2004). Eine Klassifikation mathematischer Aufgaben zur Analyse von Unterrichtssituationen. Selbsttätiges Arbeiten in Schülerarbeitsphasen in den Stunden der TIMSS-Video-Studie. Hildesheim: Franzbecker.

Norman, D. A., \& Rumelhart, D. E. (1978). Strukturen des Wissens. Wege der Kognitionsforschung. Stuttgart: Klett-Cotta.

Pauli, C., \& Reusser, K. (2006). Von international vergleichenden Video Surveys zur videobasierten Unterrichtsforschung und -entwicklung. Zeitschrift für Pädagogik, 52(6), 774-798.

Pauli, C., \& Reusser, K. (2018). Unterrichtsgespräche führen - das Transversale und das Fachliche einer didaktischen Kernkompetenz. Beiträge zur Lehrerinnen- und Lehrerbildung, 36(3), 365-377.

Praetorius, A.-K., Pauli, C., Reusser, K., Rakoczy, K., \& Klieme, E. (2014). One lesson is all you need? Stability of instructional quality across lessons. Learning and Instruction, 31, 2-12. https://doi.org/ 10.1016/j.learninstruc.2013.12.002.

Praetorius, A.-K., Herrmann, C., Gerlach, E., Zülsdorf-Kersting, M., Heinitz, B., \& Nehring, A. (2020a). Unterrichtsqualität in den Fachdidaktiken im deutschsprachigen Raum - zwischen Generik und Fachspezifik. Unterrichtswissenschaft, 48(3), 409-446. https://doi.org/10.1007/s42010-020-00082-8.

Praetorius, A.-K., Rogh, W., \& Kleickmann, T. (2020b). Blinde Flecken des Modells der drei Basisdimensionen von Unterrichtsqualität? Das Modell im Spiegel einer internationalen Synthese von Merkmalen der Unterrichtsqualität. Unterrichtswissenschaft, 48(3), 303-318. https://doi.org/10.1007/s42010020-00072-w.

Prediger, S., Link, M., Hinz, R., Hussmann, S., Thiele, J., \& Ralle, B. (2012). Lehr-Lernprozesse initiieren und erforschen - fachdidaktische Entwicklungsforschung im Dortmunder Modell. $M N U, 65(8)$, $452-457$.

Resnick, L.B., Asterhan, C.S.C., Clarke, S. N., \& Schantz, F. (2018). Next generation research in dialogic learning. In G.E. Hall, L.F. Quinn \& D. M. Gollnick (Hrsg.), Wiley Handbook of teaching and learning (S. 323-338). New York: Wiley.

Reusser, K. (1998). Denkstrukturen und Wissenserwerb in der Ontogenese. In F. Klix \& H. Spada (Hrsg.), Enzyklopädie der Psychologie. Themenbereich C: Theorie und Forschung. Serie II: Kognition. G:Wissenspsychologie. (S. 115-166). Göttingen: Hogrefe.

Reusser, K. (2008). Empirisch fundierte Didaktik - didaktisch fundierte Unterrichtsforschung. Eine Perspektive zur Neuorientierung der Allgemeinen Didaktik. In M. A. Meyer, M. Prenzel \& S. Hellekamps (Hrsg.), Perspektiven der Didaktik. Zeitschrift für Erziehungwissenschaft, Sonderheft 9/2008. (S. 219-237). Wiesbaden: VS.

Reusser, K. (2018a). Allgemeine Didaktik - quo vadis? Beiträge zur Lehrerinnen- und Lehrerbildung, 36(3), 311-328.

Reusser, K. (2018b). „Kognitive Empathie“ als Prozessmerkmal und berufsethische Qualität guten Unterrichts. Perspektiven und Anwendungen. In H. R. Schärer \& M. Zutavern (Hrsg.), Das professionelle Ethos von Lehrerinnen und Lehrern (S. 73-90). Münster: Waxmann. 
Reusser, K. (2020). Unterrichtsqualität zwischen empirisch-analytischer Forschung und pädagogisch-didaktischer Theorie. Ein Kommentar. Zeitschrift für Pädagogik, 66. Beiheft, 236-254.

Reusser, K., \& Pauli, C. (2013). Verständnisorientierung in Mathematikstunden erfassen - Ergebnisse eines methodenintegrativen Ansatzes. Zeitschrift für Pädagogik, 59(3), 308-335.

Schoenfeld, A.H. (1985). Mathematical problem solving. Orlando: Academic Press.

Seidel, T., \& Shavelson, R. J. (2007). Teaching effectiveness research in the past decade: the role of theory and research design in disentangling meta-analysis results. Review of Educational Research, 77(4), 454-499.

Selz, O. (1913). Über die Gesetze des geordneten Denkverlaufs. Erster Teil. Eine experimentelle Untersuchung. Stuttgart: W. Spemann.

Shulman, L.S. (1986). Those who understand: Knowledge growth in teaching. Educational Researcher, $15(2), 4-14 / 21$.

Shulman, L.S. (1987). Knowledge and teaching: foundations of the new reform. Harward Educational Review, 57(1), 1-22.

Staub, F.C. (2001). Fachspezifisch-pädagogisches Coaching: Theoriebezogene Unterrichtsentwicklung zur Förderung von Unterrichtsexpertise. Beiträge zur Lehrerbildung, 19(2), 175-198.

Stigler, J. W., Gallimore, R., \& Hiebert, J. (2000). Using video surveys to compare classrooms and teaching across cultures: Examples and lessons from the TIMSS video studies. Educational Psychologist, 35(2), 87-100.

Tausch, R., \& Tausch, A. (1970). Erziehungspsychologie. Begegnung von Person zu Person. Göttingen: Hogrefe.

William, D. (2015). Die Funktion der formativen Evaluation für eine erfolgreiche Lernumgebung. In H. Dumont, D. Istance \& F. Benavides (Hrsg.), Die Natur des Lernens (S. 141-162). Weinheim: Beltz.

Zimmermann, M. (2020). Dialogische Klassengesprächsführung im Geschichtsunterricht. Entwicklung einer fachlichen und transversalen Kompetenz von Lehrpersonen im Rahmen der Interventionsstudie Socrates 2.0. Dissertation. Freiburg/Fribourg: Universität Freiburg.

Zülsdorf-Kersting, M. (2020). Qualitätsmerkmale von Geschichtsunterricht. Zum Verhältnis generischer und fachspezifischer Merkmale. Unterrichtswissenschaft, 48(3), 385-407. https://doi.org/10.1007/ s42010-020-00073-9. 